REVISTA CIENCIAS BIOMÉDICAS

PRESENTACIÓN DE CASOS CLÍNICOS

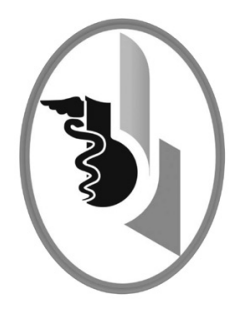

\title{
HAMARTOMA MESENQUIMATOSO HEPÁTICO GIGANTE
}

\author{
GIANT HEPATHIC MESENCHYMAL HAMARTOMA
}

\author{
Caicedo-Caicedo Luis Alfonso ${ }^{1}$ \\ Guevara-Flórez Lissette Lorena² \\ Marsiglia-Armella Heidy ${ }^{3}$ \\ Jaimes-Gutiérrez Mario ${ }^{4}$
}

Correspondencia: luisalfonso2105@gmail.com

Recibido para evaluación: octubre - 25 -2016. Aceptado para publicación: abril - 28 - 2016.

\section{RESUMEN}

Introducción: el hamartoma mesenquimatoso hepático $(\mathrm{HMH})$ es un tumor poco frecuente en la infancia, cuya etiología está relacionada con alteraciones del desarrollo embrionario del hígado.

Caso clínico: se describe un caso de $\mathrm{HMH}$ en una lactante mayor, con imágenes diagnósticas que sugerían hepatoblastoma. Se realizó biopsia hepática demostrando $\mathrm{HMH}$, se descartaron patologías malignas y se realizó resección completa del tumor. La paciente evolucionó satisfactoriamente.

Conclusión: en el diagnóstico diferencial del HMH hay que considerar el hepatoblastoma, en especial cuando se presenta con pobre componente quístico. La ecografía, la tomografía de abdomen y en especial la resonancia magnética son herramientas diagnósticas; la resección operatoria es el tratamiento adecuado. Rev.cienc.biomed. 2016;7(1):128-132.

\section{PALABRAS CLAVE}

Hamartoma; Neoplasias hepáticas; Mesenquimatoso.

\section{SUMMARY}

Introduction: hepathic mesenchymal harmatoma is an uncommon tumor during the chilhood whose etiology is related to alterations in the embryonic development of the liver.

Case report: it is described a hepathic mesenchymal hamartoma case in a female older infant with images that suggested hepatoblastoma. It was carried out a liver biopsy to show hepathic mesenchymal hamartoma to reject malign pathologies and complete resection of the tumor. The patient made a satisfactory recovery.

Médico. Estudiante de Postgrado. Pediatría. Facultad de Medicina. Universidad de Cartagena. Cartagena. Colombia.

Médico. Especialista en Pediatría. Universidad de Cartagena. Cartagena. Colombia.

Médico. Especialista en Pediatría Oncológica. Hospital Napoleón Franco Pareja. Cartagena. Colombia.

Médico. Cirujano Pediatra. Hospital Infantil Napoleón Franco Pareja. Cartagena. Colombia. 
ISSN: 2215-7840,7(1), enero-junio 2016, Caicedo-Caicedo Luis Alfonso, Guevara-Flórez Lissette Lorena, Marsiglia-Armella Heidy,

Conclusion: the hepatoblastoma should be considered in the differential diagnosis of hepathic mesenchymal hamartoma, specially when it comes with poor cystic component. The ultrasound, abdomen tomography and magnetic resonance spectroscopy are diagnostic tools; resection is the appropriate treatment. Rev.cienc. biomed. 2016;7(1):128-132.

\section{KEYWORDS}

Hamartoma; Liver neoplasms; Mesenchymal.

\section{INTRODUCCIÓN}

Los tumores hepáticos son infrecuentes y en su mayoría son de naturaleza maligna. Sin embargo, durante la lactancia, las lesiones que se identifican suelen ser benignas. Corresponden, en primer lugar, a tumores vasculares como el hemangioendotelioma infantil y en segundo lugar, al hamartoma mesenquimatoso (1).

El hamartoma mesenquimatoso hepático (HMH) corresponde al $8 \%$ de todos los tumores de la infancia (2). El $80 \%$ se presentan antes de los dos años de edad, con media de presentación a los veinte meses y predominio en el sexo masculino. El objetivo fue presentar un caso clínico de $\mathrm{HMH}$ gigante diagnosticado en el Hospital Infantil Napoleón Franco Pareja (HINFP) en Cartagena-Colombia, previo consentimiento y aprobación del Centro de Investigación y Docencia de dicha institución.

\section{CASO CLÍNICO}

Lactante de 21 meses de edad, de sexo femenino, quien ingresó al HINFP en Cartagena-Colombia, remitida de la ciudad de Santa Marta (también en el Caribe colombiano) por cuadro clínico de un mes de evolución caracterizado por sensación de masa en abdomen superior, no dolorosa, asociada a pérdida de peso de aproximadamente dos kilogramos.

En el examen físico se encontró paciente en buen estado general y nutricional. Se palpó masa de aproximadamente $8 \times 7$ centímetros que comprometía hipocondrio y flanco derecho, de consistencia dura, lisa, no dolorosa y sin movilidad.

La paciente mostró unos estudios realizados en forma ambulatoria entre los que se destacaban hemograma y función hepática normal, antígeno carcinoembrionario negativo, ecografía de abdomen que reportó masa sólida, redondeada, que comprometía el lóbulo hepático izquierdo y parte de lóbulo derecho, 68×53 mm de diámetro con áreas anecoicas en su interior y que rechazaba vesícula biliar.

Se realizó TAC abdominal que reportó masa hipodensa heterogénea, de aparente origen en el lóbulo caudado de $69 \times 77 \times 80 \mathrm{~mm}$ con necrosis central, con menor realce que el parénquima adyacente, sin invasión de la arteria hepática.

Vena porta desplazada, lo mismo en las ramas vasculares izquierdas. Lesión altamente sugestiva de hepatoblastoma. Se realizó RNM de abdomen que reportó neoplasia intrabdominal dependiente de lóbulo caudado del hígado, altamente sugestiva de neoplasia maligna (Figura No 1 y No 2).

Se realizó biopsia hepática por laparoscopia y se obtuvo muestra insuficiente. El estudio histopatológico fue concluyente, por lo que se realizó biopsia abierta en la que se observó tumor de hígado de aproximadamente diez centímetros de diámetro, vascularizado, de superficie lisa y consistencia gomosa, que tenía su origen en el lóbulo hepático izquierdo, segmento III, con crecimiento caudal, que llegaba a fosa ilíaca derecha. El estudio histopatológico reportó HMH gigante. No se señalaron criterios histológicos de malignidad.

Se adelantó TAC de tórax simple y contrastado que reportó presencia de engrosamiento pleural derecho, sin adenopatías. Dosificación de alfa fetoproteína dentro de límites normales. Se realizó laparotomía exploratoria y se extrajo la totalidad del tumor (Figu- 


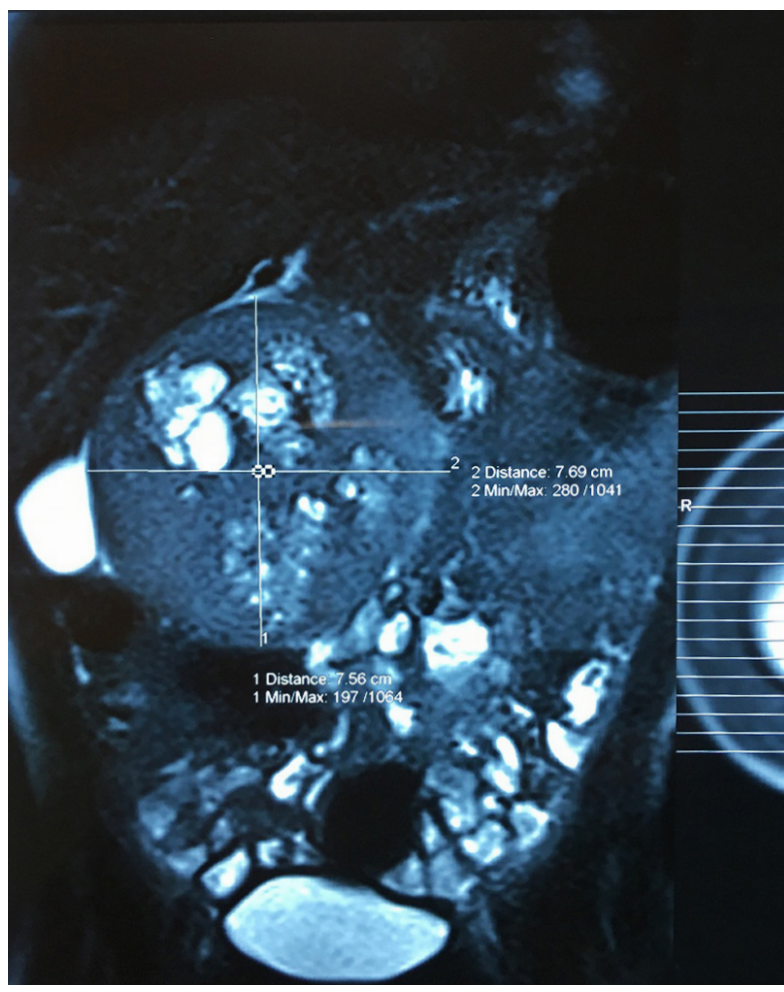

Figura $\mathbf{N}^{0} 1$.

Corte axial en RMN de abdomen que muestra tumor con efecto compresivo sobre estructuras adyacentes.

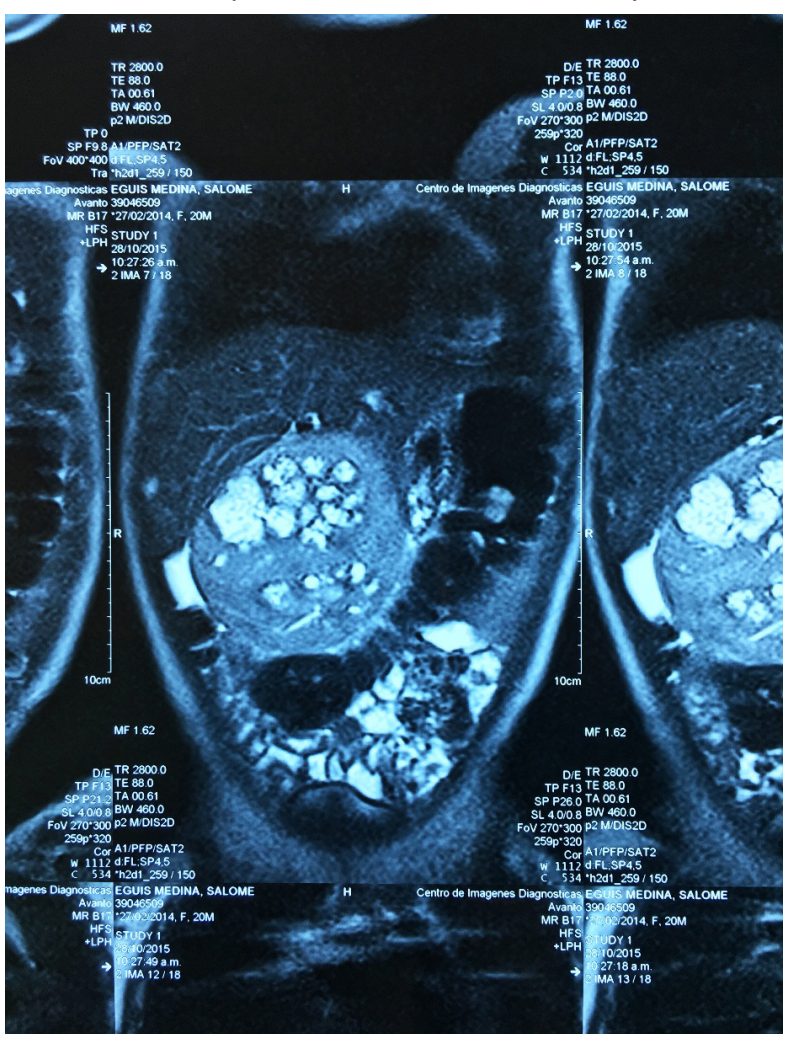

Figura $\mathbf{N}^{0} \mathbf{2}$

Corte coronal en RMN de abdomen que muestra tumor en relación estrecha con cara inferior del hígado. ras $\mathrm{N}^{0}$ 3, 4 y 5). El estudio histopatológico y de inmunohistoquímica confirmó el diagnóstico previo de HMH. La paciente evolucionó satisfactoriamente y ha permanecido asintomática en su seguimiento ambulatorio.

\section{DISCUSIÓN}

Los tumores primarios de hígado constituyen menos del $3 \%$ de los tumores observados en la población pediátrica, y solo un tercio de estos tumores son benignos. Los tumores benignos pueden ser epiteliales: (hiperplasia nodular focal, adenoma hepatocelular), mesenquimal (hemangioma hepático, hamartoma mesenquimal), u otros (teratoma, pseudotumor inflamatorio) (3).

El hamartoma mesenquimatoso hepático se presenta principalmente en menores de 2 años. Es el tercer tumor más frecuente del hígado en este grupo de edad (después de hepatoblastoma y hemangioma infantil), representando aproximadamente el 6\% de todos los tumores primarios del hígado (4-6). Se describe un predominio de presentación en pacientes masculinos con una relación $2: 1$, sin asociación a raza o localización geográfica (7).

Los hamartomas resultan de la proliferación no coordinada de mesénquima primitivo en los tractos periportales y pueden ser sólidos o quísticos. Por lo general son multiquísticos, y los quistes están revestidos con epitelio biliar aplanado o endotelio $(4,5)$. En la evaluación histológica el hamartoma consiste en quistes, restos de tríadas portales, hepatocitos y el fluido del mesénquima (6).

Clínicamente, la mayoría de los $\mathrm{HMH}$ son vistos como distensión o masa abdominal como el caso que se presenta. En algunos casos pueden crecer y alcanzar un gran tamaño y causar dificultad respiratoria o evidencia de obstrucción de la vena cava (4). Para el diagnóstico el ultrasonido suele ser el estudio inicial y permite observar una masa compleja multiquística con septos internos que se pueden detectar in utero $(7,6)$. En la tomografía computarizada de abdomen (TAC) estos tumores aparecen como masas bien definidas con áreas hipodensas centrales y los tabiques internos. 


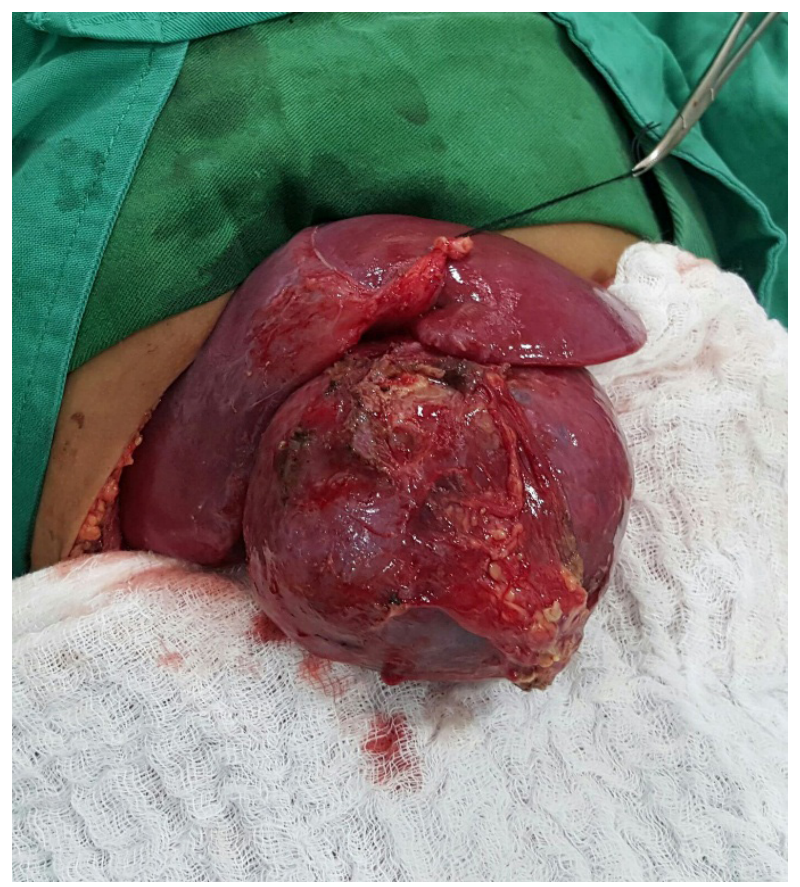

Figura $\mathbf{N}^{0} 3$.

Tumor en su aspecto macroscópico y su relación con el hígado, previo a resección quirúrgica.

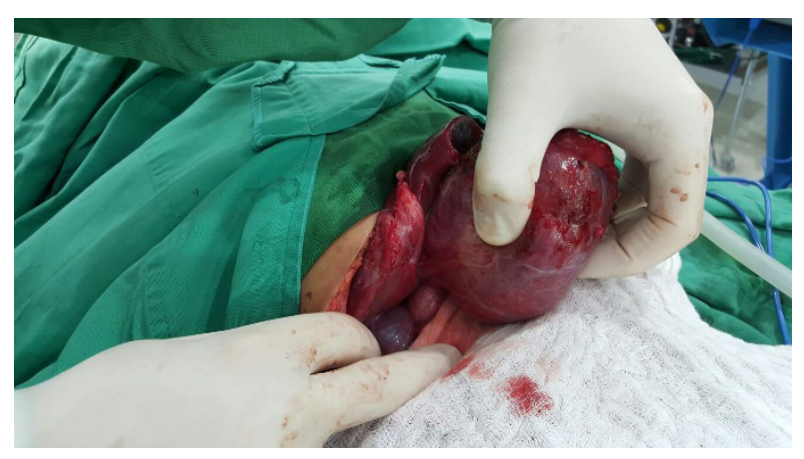

Figura $N^{0} 4$.

Maniobras de control vascular antes de la resección del tumor.

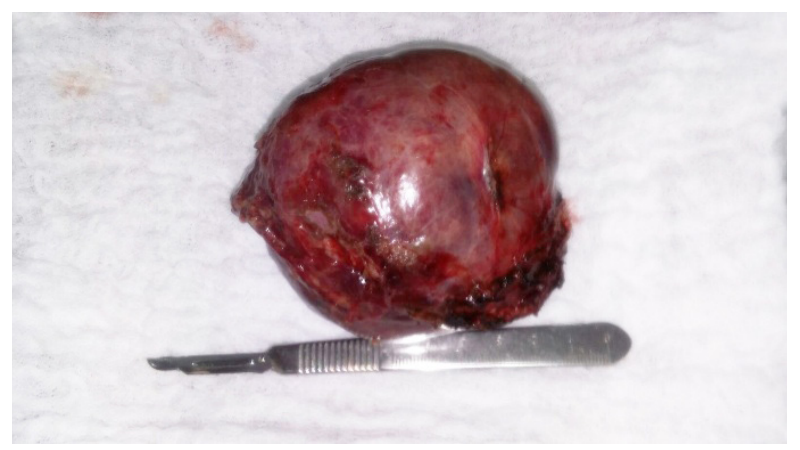

Figura $\mathbf{N}^{0} 5$.

Resección completa de la masa.
Después de la administración de medios de contraste, las áreas sólidas pueden ser detectables fácilmente. El estudio de imagen de elección es la resonancia magnética (RMN). Para las lesiones con predominio del estroma, la intensidad de las imágenes en T1 es menor que la del hígado normal debido al aumento de la fibrosis. Por el contrario, si hay predominio quístico, la masa es predominantemente hipointensa en T1 y marcadamente hiperintensa en T2. Los componentes del estroma pueden mejorar después de la administración de gadolinio (6). Una biopsia abierta es a menudo necesaria para hacer el diagnóstico (7).

Teniendo en cuenta que el principal diagnóstico diferencial en el caso que se presenta era el hepatoblastoma, resulta importante dar a conocer las diferencias entre estos dos tipos de tumores. El HMH es un tumor benigno con una distribución por edad similar al hepatoblastoma, en casi todos los casos se produce en menores de 5 años. La presentación clínica puede parecerse a la del hepatoblastoma con distensión abdominal, el nivel de alfa-fetoproteina en suero suele estar en rangos normales como en el caso de esta paciente, a diferencia del hepatoblastoma en el que se espera encontrar niveles elevados de este marcador. Al igual que con el hepatoblastoma, la mayoría de los $\mathrm{HMH}$ surgen en el lado derecho del hígado. Además, la presencia de quistes es más característica en el hamartoma que en el hepatoblastoma.

En el caso de la paciente en cuestión, las imágenes diagnósticas evidenciaron una masa sólida con poco componente quístico que hizo considerar el diagnóstico de hepatoblastoma como primera opción, antes de obtener el resultado de patología. Tanto los hamartomas como los hepatoblastomas se caracterizan por un crecimiento masivo, y causan morbilidad mediante la compresión de estructuras adyacentes. Microscópicamente los $\mathrm{HMH}$ constan de células fusiformes en un fondo mixoide con áreas ocasionales de hematopoyesis extramedular similar a la observada en hepatoblastoma (8). Citogenéticamente se ha reportado que en estos tumores existen translocaciones balanceadas entre cromosomas 11, 17 y $19(7,9,10)$. El HMH puede mostrar la 
transformación maligna en mesenquimoma maligno o sarcoma embrionario y esto podría tener una base genética $(10,11)$.

Por otra parte, se ha reportado la regresión espontánea de estos tumores, esto ha llevado a algunos autores a cuestionar la necesidad de tratamiento quirúrgico. Sin embargo, en una revisión sistemática ninguna de las lesiones regresó por completo $(12,13)$.

El tratamiento, generalmente, consiste en la extirpación quirúrgica que suele ser eficaz. No obstante, la mortalidad y morbilidad de este tipo de tumores hepáticos están primariamente relacionadas con complicaciones quirúrgicas. La mortalidad perioperatoria es relativamente alta (hasta un $17 \%$ ) y está en relación con las dificultades técnicas de la resección quirúrgica (7). A pesar de ello, con las técnicas modernas las complicaciones durante o después de la resección del tumor son cada vez menos frecuentes. Los estudios de imagen tienen un papel importante en el manejo del paciente, no solo en el diagnóstico, sino también en la definición de las dimensiones del tumor y las relaciones anatómicas, de tal manera que ayudan en la viabilidad y enfoque de la cirugía (12), por ejemplo, las lesiones pedunculadas son susceptibles de resección laparoscópica. El pronóstico es excelente luego de la resección completa del tumor y es aconsejable una revisión clínica y ultrasonográfica al menos durante cinco años (13).

\section{CONCLUSIÓN}

Dentro de los tumores hepáticos benignos el hamartoma mesenquimatoso es el segundo en frecuencia. En el diagnóstico diferencial hay que considerar el hepatoblastoma, en especial cuando se presenta con pobre componente quístico. La ecografía, la tomografía de abdomen y en especial, la resonancia magnética representan las ayudas diagnósticas más importantes; el diagnóstico definitivo se realiza mediante biopsia y estudio anatomopatológico. El tratamiento quirúrgico es curativo y tiene excelente pronóstico.

CONFLICTO DE INTERESES: ninguno que declarar.

FINANCIACIÓN: recursos propios de los autores. Estudios imagenológicos, material quirúrgico e insumos hospitalarios, así como honorarios profesionales y demás costos clínicos fueron aportados dentro de la atención asistencial.

\section{REFERENCIAS BIBLIOGRÁFICAS}

1. Vargas-Vallejo MP, Álvarez-Solís RM, Cabrera-Aguirre J, Bulnes-Mendizábal DP, Ulloa-Patiño P. Hamartoma mesenquimatoso hepático gigante en una niña de 2 años. An Pediatr.2005;63: 564-5.

2. Masood A. Siddiqui, MD; Barbara J. McKenna, MD. Hepatic Mesenchymal Hamartoma, A Short Review. Arch Pathol Lab Med. 2006;130: 1567-69.

3. Wolfgang S, Philip G. Nonmalignant tumors of the liver. In: Arnold G. Coran. Pediatric Surgery. Seventh Edition. 2012;32: 459-62.

4. Rich-Barrie S, La Q, Michael P. Hepatic tumors in childhood. In: Jarnagin, William R., MD, FACS. Blumgart's surgery of the liver, biliary tract and pancreas. Fifth Edition. 2012;82:132-43.

5. Linda F. Benign and malignant tumors of the liver. In: Odze and Goldblum Surgical Pathology of the GI Tract, Liver, Biliary Tract and Pancreas. Third Edition. 2015;55:1539-72.

6. Pablo RR, Sukru ME. Benign tumors of the liver. In: Richard M. Gore MD, Marc S. Levine MD. Textbook of Gastrointestinal Radiology. Fourth Edition. 2015; 86:1528-60.

7. Klein-Moreira R, Washington K. Liver neoplasms. In: Christine A. Lacobuzio-Donahue and Elizabeth Montgomery. Gastrointestinal and liver pathology. Second edition. 2012;20:626-77.

8. Tomlinson GE. Hepatoblastoma and other liver tumors in children. In: Nathan and Oski's Hematology and Oncology of Infancy and Childhood. Eighth Edition. 2015;58:1886-1905.

9. Murthi GV, Paterson L, Azmy A. Chromosomal translocation in mesenchymal hamartoma of liver: what is its significance? Journal of Pediatric Surgery. 2003; 38(10):1543-45.

10. Mathews J, Eric J. Duncavage JD. Pfeifer. Characterization of translocations in mesenchymal hamartoma and undifferentiated embryonal sarcoma of the liver. Experimental and molecular pathology. 2013; 95:319-24.

11. Ramanujam JC, Ramesh DW, Goh KT, Wong W.A. Ariffin, G. Kumar, and N.A. Taib. Malignant transformation of mesenchymal hamartoma of the liver: case report and review of the literature. Journal of Pediatric Surgery.1999;34(11):1684-86.

12. G Nair, M Fortier and Y Low. Cystic hepatic mesenchymal hamartoma: the role of radiology in diagnosis and perioperative management. The British Journal of Radiology. 2011;84:91-4.

13. Mark D. Stringer, Naved K. Alizai. Mesenchymal hamartoma of the liver: a systematic review. Journal of Pediatric Surgery.2005:40:1681-90. 